\title{
Correction to: Measurement of Sheet-like Space Charge Distribution and Signal Calibration of Insulator XLPE Using PEA Method
}

\author{
Hyung-Gyu Kim ${ }^{1} \mathbb{D} \cdot$ Hyun-Cheol Jung ${ }^{1,2} \cdot$ June-Do Park $^{1,2}$. Seung Hwangbo ${ }^{1}$
}

Published online: 19 November 2020

(c) The Korean Institute of Electrical Engineers 2020

\section{Correction to: \\ Journal of Electrical Engineering \& Technology \\ https://doi.org/10.1007/s42835-020-00477-8}

Unfortunately the acknowledgement has been omitted. It should be: This work was supported by the KEPCO grant \#R17XA05-03 and by the Smart Grid Development of Core Technology Project of the KETEP grant funded by MOTIE, Korea NO. 20171210201080.

The original article can be found online at https://doi.org/10.1007/ s42835-020-00477-8.

Seung Hwangbo

hbs@honam.ac.kr

Hyung-Gyu Kim

khg8532@gmail.com

Hyun-Cheol Jung

jhc8102@naver.com

June-Do Park

junedo.park@nisquare.co.kr

1 Department of Automotive Engineering, Honam University, Gwangju 62399, Republic of Korea

2 Department of Electrical and Electronic Engineering, Honam University, Gwangju 62399, Republic of Korea 